\title{
Transoral endoscopic excision without robots or lasers: a novel diagnostic and therapeutic approach to base of tongue tumours in two patients
}

\begin{abstract}
Objective: The purpose of this article is to illustrate the use of transoral endoscopic resection, without robots or lasers, in the management of base of tongue tumours as an alternative to other existing techniques.

Case reports: Two cases of tongue base tumours were excised using transoral endoscopic approach with blade and diathermy. Both patients were able to tolerate oral intake with successful decannulation of their tracheostomies post-operatively. The first tumour turned out to be an ectopic thyroid while the second tumour was found to be a mucoepidermoid carcinoma.

Discussion and conclusion: This approach is minimally invasive compared to open surgeries with early return of normal swallowing and speech post-operatively. It also offers diagnostic benefit for submucosal tumours and does not require additional training or funding for expensive new equipment. We find this method to be of considerable clinical usefulness in the management of base of tongue tumours.
\end{abstract}

Volume 9 Issue 3 - 2017

\begin{abstract}
Eugene Hung Chih Wong, Hui Tong Wong, Abu Bakar Zulkiflee, Narayanan Prepageran ENT Department University Malaya Medical Centre, Malaysia
\end{abstract}

Correspondence: Eugene HCWong Otorhinolaryngology ENT Department University Malaya Medical Centre Jalan University Kuala Lumpur 59100, Malaysia, Tel +60126395363, Email Eugene.wong.hc@gmail.com

Received: October 16, 2017 | Published: December 08, 2017

Keywords: tongue, base, tumour, endoscopic, transoral

Abbreviations: BOT, base of tongue; HPV, human papillomavirus; TORS, transoral robotic Surgery; CRT, concurrent chemotherapy; NG, nasogastric tubes; FESS, functional endoscopic sinus surgeries

\section{Introduction}

The incidence of base of tongue tumours (BOT) has risen significantly over the last few decades due to an epidemic of the human papillomavirus (HPV). ${ }^{1}$ The management options for BOT have been controversial where conventionally; patients were treated with either surgery, radio-chemotherapy or various combinations of these options. ${ }^{2}$

Transoral laser microsurgery and transoral robotic surgery (TORS) resection of BOTs were introduced to provide better functional outcome in patients post-operatively, while maintaining good oncological outcome. ${ }^{2,3}$ We report two cases of tongue base tumours that were excised using transoral endoscopic approach without robots or lasers. We have highlighted the specific advantages of this technique in certain clinical situations.

\section{Case reports}

\section{Case I}

An eighteen year-old girl presented with 5months history of hot potato voice, dyspnoea and dysphagia. On examination, there was a base of tongue mass occupying the oral cavity, obstructing the airway. MRI showed a well-defined BOT mass displacing the epiglottis posteriorly, occupying the entire oropharynx and valleculae, with feeding vessels from both lingual arteries (Figure 1).

She underwent elective tracheostomy to secure the airway, followed by embolization of the feeding vessels (sublingual arteries) before proceeding to transoral endoscopic excision of the base of tongue mass (Figure 2) using blade and diathermy. She was able to tolerate oral intake at day four post-operatively. She also had a good airway after the operation and her tracheostomy was successfully decannulated four weeks after the operation with return of normal speech. Histological examination confirmed the mass to be an ectopic thyroid with adenomatous proliferation. She was hypothyroid postoperatively and was subsequently started on Thyroxine.

\section{Case 2}

A seventeen-year-old girl presented with four months history of progressively worsening hoarseness, dysphagia, bilateral neck swelling and shortness of breath with intermittent stridor. Examination revealed a base of tongue mass obstructing the airway with bilateral level three and four cervical lymphadenopathy. MRI demonstrated an ill-defined lobulated heterogenous BOT mass obstructing the airway (Figure 3) with bilateral cervical lymphadenopathy.

Elective local anaesthetic tracheostomy was performed for airway protection followed by removal tongue base mass via transoral endoscopic resection under general anaesthetic (Figure 4) using blade and diathermy. She had good airway post-operatively and was able to tolerate oral feeding five days after the operation. Six weeks after the operation, her tracheostomy was successfully decannulated with return of normal speech function. Histological examination concluded the mass to be a mucoepidermoid carcinoma. She was subsequently treated with chemoradiotherapy.

\section{Discussion}

The National Comprehensive Cancer Network (NCCN) Guidelines $(2012)^{4}$ recommended usage of surgery or radiotherapy (RT) or radiotherapy with concurrent chemotherapy (CRT) in the treatment of patients with early stage oropharyngeal cancers. However, open surgeries to the oropharynx have been associated with poorer quality of life ${ }^{5}$ due to morbidities such as malocclusion, dysphagia and cosmetic deformity. ${ }^{3}$ Similarly, organ-preserving treatments (RT or 
CRT) have also been found to cause functional impairments such as severe long-term dysphagia with dependence on gastrostomy tube. ${ }^{6}$

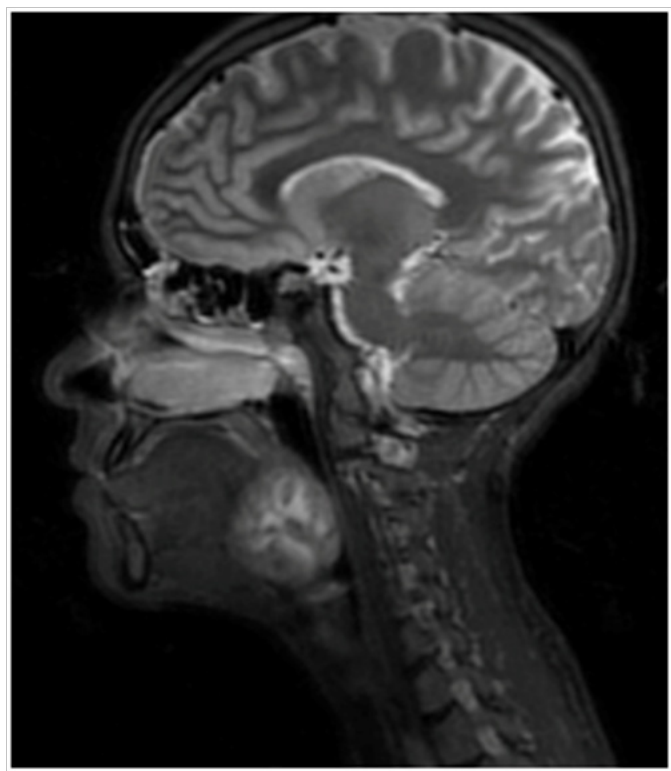

Figure I MRI imaging for Case I.
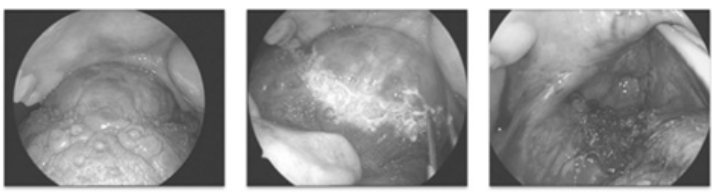

Figure 2 Intra-operative view for Case I.

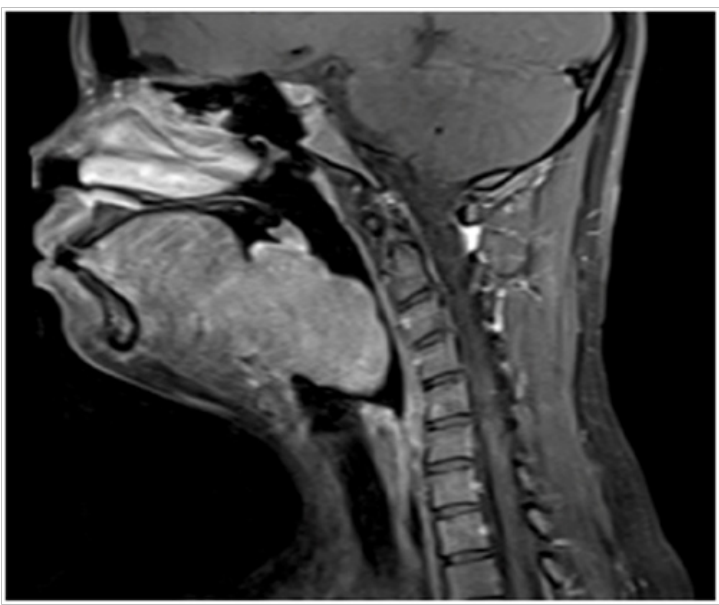

Figure $3 \mathrm{MRI}$ imaging for Case 2.
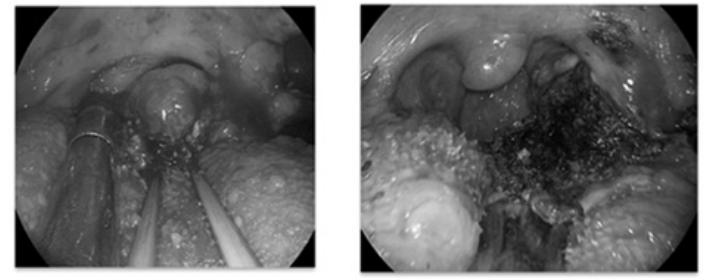

Figure 4 Intra-operative view for Case 2.
Steiner et al., ${ }^{2}$ introduced transoral laser microsurgery in 2003 for removal of base of tongue carcinoma as an alternative to the conventional open approach and found good oncological and functional outcome in patients post-operatively.

In 2006, O'Malley et al., ${ }^{7}$ described transoral robotic surgery (TORS) approach for tongue base tumours where they found this method to be superior over open surgery or transoral laser surgery as it provides excellent three-dimensional visualization and instrument access, allowing successful complete resections of tumour and excellent haemostasis. There were also no significant subjective changes in patients' self-perceived voice, swallowing and quality of life status one year after surgery. ${ }^{3}$ However, various disadvantages of TORS have also been identified such as lack of tactile feedback for surgeons and the high cost of purchasing and maintaining the robot and its instruments. ${ }^{8}$ Besides that, specific training in operating room preparation and analysis of specimens obtained using robotic surgery is also required for operating room nurses and pathologists respectively. ${ }^{8}$

We performed excision of two tongue base tumours using transoral endoscopic approach with blades and diathermy, without the need for robotic system or lasers. So far, there have been no other literatures found describing this method being used for BOTs.

We find this method to be of considerable clinical usefulness for various reasons. First, it is minimally invasive compared to open surgeries with good post-operative functional outcome. Both patients had tracheostomy prior to the excision to protect the airway. They were both decannulated four to six weeks post-operatively, after confirmation of safe airway using flexible nasendoscopy, with return of normal speech. Nasogastric tubes (NG) were placed in both patients to avoid aspiration in the first post-operative period. Both patients had their NG tubes removed four to five days post-operatively after clinical examinations confirmed that they could tolerate oral diet without risk of aspiration.

A commonly described clinical problem for tongue base tumours that grow into the deep tissue planes underneath an intact mucous membrane is the repeated false-negative results from conventional needle biopsies, causing delay in decision-making and initiation of treatment, therefore worsen patients' prognosis. ${ }^{9}$ From clinical examination, both tumours appeared to be submucosal, therefore we proceeded to transoral endoscopic excision as part of the initial diagnostic process. Both patients had definitive histopathological results where first patient with benign tumour avoided a second procedure and the second patient was able to proceed to chemoradiotherapy in a timely manner.

Finally, robots and lasers may not be readily available in many hospitals due to lack of funding or expertise in their usage. On the other hand, most otorhinolaryngologists are trained in endoscopic operations such as functional endoscopic sinus surgeries (FESS). Besides that, this method also allows good tactile feedback to surgeons during tissue handling.

\section{Conclusion}

There is a clinical role for transoral endoscopic approach for tongue base tumours, which serves as both diagnostic and therapeutic procedure, with good functional outcome.

\section{Presentation}

This paper has not been presented in any meetings. 


\section{Disclosure of interest}

The authors declare that they have no conflicts of interest concerning this article.

\section{Financial support or funding}

No financial support or funding received for this article.

\section{Acknowledgments}

None.

\section{References}

1. Gillison ML, Koch WM, Capone RB, et al. Evidence for a causal association between human papillomavirus and a subset of head and neck cancers. J Natl Cancer Inst. 2000;92(9):709-720.

2. Steiner $\mathrm{W}$, Fierek $\mathrm{O}, \mathrm{Ambrosch} \mathrm{P}$, et al. Transoral laser microsurgery for squamous cell carcinoma of the base of the tongue. Arch Otolaryngol Head Neck Surg. 2003;129(1):36-43.

3. Mercante G, Masiello A, Sperduti I, et al. Quality of life and functional evaluation in patients with tongue base tumors treated exclusively with transoral robotic surgery: A 1-year follow-up study. J Craniomaxillofac Surg. 2015;43(8):1561-1566.
4. Guidelines N. Clinical practice guidelines in oncology: head and neck cancer. 2012

5. Zhen W, Karnell LH, Hoffman HT, et al. The National Cancer Data Base report on squamous cell carcinoma of the base of tongue. Head Neck. 2004;26(8):660-674.

6. Caudell JJ, Schaner PE, Meredith RF, et al. Factors associated with longterm dysphagia after definitive radiotherapy for locally advanced headand-neck cancer. Int J Radiat Oncol Biol Phys. 2009;73(2):410-415.

7. O'Malley BW, Weinstein GS, Snyder W, et al. Transoral robotic surgery (TORS) for base of tongue neoplasms. Laryngoscope. 2006;116(8):1465-1472.

8. Hans S, Delas B, Gorphe P. Transoral robotic surgery in head and neck cancer. Eur Ann Otorhinolaryngol Head Neck Dis. 2012;129:32-37.

9. Pfeiffer J, Maier W, Boedeker CC. Transmucosal core needle biopsy: A novel diagnostic approach to oral and oropharyngeal lesions. J Oral Maxillofac Surg . 2014;72(8):1594-1600. 